\title{
Estrutura interna do Myers Briggs Type Indicator (MBTI): evidência de validade
}

\author{
Gleiber Couto ${ }^{1}$ \\ Universidade Federal de Goiás, Catalão-GO, Brasil \\ Daniel Bartholomeu, José Maria Montiel \\ Centro Universitário FIEO, Osasco-SP, Brasil
}

\section{RESUMO}

Este estudo teve como objetivo analisar a estrutura fatorial do MBTI. Para tanto, foi utilizado um banco de dados contendo descrições e dados para o MBTI coletados entre 2006 e 2009 e perfazendo um total de 6.460 protocolos de sujeitos com idades variando entre 14 e 77 anos. Foram realizados dois estudos. Na etapa 1, os resultados demonstraram que todos os itens se agrupam adequadamente para medir a dimensão psicológica em questão. Já na etapa 2, para a realização da Análise Fatorial Confirmatória, a amostra foi composta por 5190 participantes com idades variando entre 18 e 77 anos. Os resultados dessa etapa apontaram para quatro fatores independentes, funções de Julgamento, Atitudes, Percepção e Orientação, reunindo todos os 93 itens da versão brasileira da escala. Tais achados, nas duas etapas, demonstram serem condizentes com as expectativas teóricas.

Palavras-chave: evidências de validade, psicometria, personalidade.

\section{ABSTRACT - Internal structure of the Myers Briggs Type Indicator (MBTI): Evidence of validity}

This study aimed to examine the factor structure of the MBTI. To this end, a database was used which contained descriptions and data for the MBTI, collected between 2006 and 2009 with a total of 6,460 protocols, for subjects aged between 14 and 77 years. Two studies were conducted. In Step 1, the results showed that all items are properly grouped to measure the psychological dimension in question. In Step 2, to perform the Confirmatory Factor Analysis, the sample consisted of 5,190 participants aged between 18 and 77 years. The results of this step pointed to four independent factors, functions of Judgment, Attitudes, Perception and Orientation, including all 93 items of the Brazilian version of the scale. These findings demonstrate the two steps are consistent with theoretical expectations. Keywords: validity evidence; psychometrics; personality.

\section{RESUMEN - Estructura interna del Myers Briggs Type Indicador (MBTI): evidencia de validez}

Este estudio tuvo como objetivo analizar la estructura factorial del MBTI. Para ello, fue utilizada una base de datos que contiene descripciones y datos, recogidos entre 2006 y 2009, completando un total de 6.460 protocolos de sujetos con edades comprendidas entre 14 y 77 años. Se realizaron dos estudios. En la primer etapa, los resultados mostraron que todos los ítems se agrupan adecuadamente para medir la dimensión psicológica en cuestión. Ya en la segunda etapa para realizar el Análisis Factorial Confirmatorio, la muestra estuvo formada por 5.190 participantes, con edades entre 18 y 77 años. Los resultados de esta etapa señalaron cuatro factores independientes: Funciones de juicio, Actitudes, Percepción y Orientación, reuniendo los 93 ítems de la versión brasileña de la escala. Los resultados en las dos etapas demuestran concordar con las expectativas teóricas.

Palabras clave: Evidencias de validez; Psicometría; Personalidad.

A Psicologia Analítica de Jung sempre teve em seu horizonte epistemológico a investigação dos modos como cada pessoa constitui sua singularidade a partir de uma base comum a toda a humanidade: o inconsciente coletivo (Jung, 1928/1971; Jung, 1964). Nessa perspectiva, entende-se que as pessoas se desenvolvem a partir da interação complexa de fatores hereditários e ambientais, sendo que a singularidade representa a maneira única como cada indivíduo se constitui subjetivamente em seu meio. Por outro lado, a doutrina da singularidade não oferece subsídios práticos para compreender as pessoas em contextos coletivos: na educação, no aconselhamento, nas rotinas de trabalho e na vida social. Na prática, as pessoas tendem a assumir inconscientemente que os seus modos e estilos de funcionamento consciente sejam iguais aos das outras pessoas com quem se relacionam (Pasquali, 2003). A ideia de que exista uma similaridade universal entre o modo como os indivíduos percebem 
e avaliam a realidade pode gerar inúmeros equívocos quanto aos estilos de comportamento de pessoas cujos padrões arquetípicos de funcionamento mental são muito diferentes (Bartholomeu, Montiel, \& Bernstein, 2014; Bartholomeu, Montiel, \& Pessotto, 2012; Bento, Bartholomeu, Montiel, \& Machado 2013).

Segundo Zacharias (1995), inicialmente, a proposta de Jung foi formular hipóteses sobre diferenças individuais de personalidade a partir da observação de dois tipos básicos de comportamento: a extroversão e a introversão. Ele identificou os tipos extrovertidos como indivíduos cuja energia psíquica tende a se dirigir para fora, isto é, para pessoas e eventos do ambiente externo. Por outro lado, o tipo introvertido tem sua energia psíquica orientada para dentro e sua atenção dirige-se aos pensamentos e experiências do ambiente interno. Nos postulados de Jung (1928/1971) é possível observar que o autor subdividiu os tipos extrovertidos e introvertidos em oito tipos psicológicos, por meio da identificação de dois pares opostos de atitudes da consciência, as funções de Percepção - sensação e intuição, e as funções de Julgamento - pensamento e sentimento.

Jung (1928/1971) define o termo "funções" como "uma forma específica de atividade psíquica que por princípio permanece inalterada mesmo sob condições variadas". As funções de Percepção referem-se à aquisição de informações, ao passo que as funções de Julgamento referem-se à maneira como o indivíduo chega a conclusões a respeito daquilo que é percebido. Também estabeleceu relações entre as atitudes mais habituais da consciência (extroversão ou introversão) e as funções ditas dominantes de um indivíduo. O termo "função dominante" refere-se ao processo mental, sensação, intuição, pensamento ou sentimento, que uma pessoa utilizará mais frequentemente, de maneira mais natural e confiável. A função dominante pode ser considerada como aquela que orienta ou predomina na personalidade consciente.

A elaboração conceitual desses dois pares de processos mentais, em adição a par de atitudes inicialmente proposto levou à formulação de oito combinações tipológicas (Jung, 1928/1971). A primeira foi sensação extrovertida, energia psíquica orientada para o ambiente externo, informações adquiridas pela atenção aos detalhes e apreensão de dados sensoriais com precisão e foco no momento presente. A segunda, sensação introvertida, energia psíquica orientada para o ambiente interno, apreensão de fatos e detalhes tanto da realidade externa quanto de pensamentos e experiências internas. A terceira foi intuição extrovertida, energia psíquica orientada para o ambiente externo, apreensão de novas ideias, foco em padrões de relação entre fatos, dados da realidade e possibilidades futuras. A quarta, intuição introvertida, energia psíquica orientada para o ambiente interno, apreensão de imagens do inconsciente e de padrões de relação entre fatos, capacidade de insight. A quinta, pensamento extrovertido, busca de ordenamento lógico para o ambiente externo pela análise objetiva da realidade e ações claramente direcionadas para a solução de problemas. A sexta, pensamento introvertido, busca de precisão e ordenamento dos pensamentos pela reflexão e desenvolvimento de um sistema lógico de compreensão. A sétima, sentimento extrovertido, busca de harmonia por meio da organização e estruturação do ambiente para adequar aos seus valores pessoais e atender às necessidades das outras pessoas. E a oitava, sentimento introvertido, busca de harmonia interna intensamente significativa e complexa, por meio da sensibilidade aos valores pessoais e comportamentos manifestados por si próprio e pelas outras pessoas (Lessa, 2003).

Briggs-Myers, Kirby e Myers (1998) desenvolveram, nos anos 40, um instrumento para avaliar características de personalidade, o Myers Briggs Type Indicator (MBTI), baseando-se nas formulações de Jung acerca do modo como as funções da Consciência interagem, isto é, o aspecto psicodinâmico do modelo tipológico. As autoras apresentaram um quarto eixo que foi mencionado nos trabalhos de Jung. Esse quarto eixo já pode ser visto no trabalho de Jung, porém não foi adequadamente desenvolvido. $\mathrm{O}$ quarto eixo refere-se a dois modos de orientação em relação ao mundo externo: aberto (perceptivo) ou estruturado (julgador). Essa distinção proposta pelas autoras refere-se ao funcionamento mais espontâneo do indivíduo em situações necessariamente extrovertidas. O termo "julgamento" denota um estilo de vida organizado e planejado por meio da avaliação e controle de variáveis e dados da realidade e reflete-se em modos estruturados e metódicos de se lidar com o mundo externo. Já o termo "percepção" denota uma abordagem flexível e espontânea ao lidar com dados da realidade por meio da abertura às experiências e reflete-se em modos criativos e adaptativos de se lidar com o mundo externo (Briggs-Myers et al., 1998).

A partir das combinações entre os pares de opostos em cada eixo, foram estabelecidos 16 tipos, identificados cada um por quatro letras, conforme a Tabela 1.

Tabela 1

Disposição dos Tipos na Perspectiva Junguiana

\begin{tabular}{clll}
\hline Ordem de cada letra & & Eixos e características a eles associadas \\
\hline $1^{\mathrm{a}}$ & Disposição & $\mathrm{E}$ - Extroversão & $\mathrm{I}$ - Introversão \\
$2^{\mathrm{a}}$ & Percepção & $\mathrm{S}$ - Sensação & $\mathrm{N}$ - Intuição \\
$3^{\mathrm{a}}$ & Julgamento & $\mathrm{T}$ - Pensamento & F - Sentimento \\
$4^{\mathrm{a}}$ & Função (orientação)? & $\mathrm{J}$ - Julgamento & $\mathrm{P}$ - Percepção \\
\hline
\end{tabular}


O modelo proposto pelo MBTI encontra grande aceitação entre psicólogos em diversos países, inclusive no Brasil, sendo utilizado em diversas áreas das ciências humanas, por apresentar tipologias específicas e que descrevem características de um indivíduo principalmente na área de Psicologia Organizacional, Educacional e Clínica (Folger, Kanitz, Knudsen, \& McHenry, 2003; Lessa 2003; Nagelschimidt 1993; Ramos 2005; Wheeler, Hunton, \& Bryant, 2004; Ziegert, 2000). No entanto, segundo os apontamentos já realizados por Pasquali (1997), os parâmetros psicométricos do teste devem ser analisados de maneira mais cuidadosa. Os índices de precisão giram em torno de 0,70 , e estudos independentes sobre a validade do MBTI, sobretudo em comparação com outros testes da área (validade concorrente), não têm conseguido fundamentar a contento esse parâmetro do teste. O autor chama atenção especialmente para a necessidade do teste de mostrar sua validade fatorial sem a qual a distinção em tipos psicológicos se torna bastante arbitrária.

Apesar desse alerta, em estudos que avaliam os parâmetros psicométricos do MBTI em diversos países, e em diferentes grupos dentro do mesmo país, principalmente no tocante à consistência interna dos fatores, tem-se obtido índices satisfatórios (Beuke, Freeman, \& Wang, 2006; Deakin, 2006; Hackston, 2005; Harvey, Murry, \& Stamoulis, 1995; Saggino \& Kline, 1995; Saggino, Cooper, \& Kline, 2000; Schaubhut, Herk, \& Thompson, 2009; Schaubhut \& Thompson, 2010; Taylor \& Yiannakis, 2007). Porém, a maior parte desses estudos faz parte de pesquisas de validação dos testes para sua utilização nos países onde os estudos se realizaram.

No entanto, outros autores também têm realizado uma série de críticas ao MBTI. Segundo Pittenger (1993), outra crítica feita ao modelo do MBTI se dá em relação ao seu excessivo rigor em estabelecer as classificações por meio de pontos de corte. Pessoas podem ter escores relativamente próximos, mas se estiverem em diferentes lados do ponto de corte aparecerão com perfis distintos de personalidade. Além disso, a partir de um estudo de revisão bibliográfica sobre o MBTI, o autor encontrou uma série de estudos em que as análises fatoriais apresentam resultados inconsistentes com a teoria que fundamenta o teste. Além disso, mostra que as conclusões do teste, principalmente quando usado para área ocupacional, são extremamente falhas quando submetidos a análises estatísticas mais detalhadas.

Já Gardner e Martinko (1996) em outro estudo de revisão bibliográfica sobre teste, encontram dados mais satisfatórios quanto à validade e à precisão do teste. Segundo os autores, muitos dos estudos sobre o teste apresentam problemas em função de estarem associados a estudos de validade que têm por base o autorrelato dos entrevistados, o que causaria uma série de distorções, indicando que os estudos deveriam partir de múltiplas fontes de comparações. No entanto, os dados referentes à estrutura interna fatorial do MBTI geralmente apresentaram resultados menos satisfatórios, principalmente nos grupos onde existe uma concentração pouco acentuada em escores extremos, fazendo também uma crítica à definição puramente dicotômica das características de personalidade dos sujeitos.

Dentre os usos mais atuais do MBTI, esse instrumento tem servido como base para estudos na cibernética, visando o desenvolvimento de personalidade em robôs e formatos de avaliação em celulares (Lee, Choi, \& Stonier, 2012; Rien, Marian, \& Helen, 2010; Yolles \& Fink, 2009). Também empregado para predizer sucesso em envolvimento e desenvolvimento de projetos em empresas (Cohen, Ornoy, \& Keren, 2013) Ainda, Yilmaz e O'Connor (2012) utilizaram o MBTI em um jogo para avaliar a personalidade das pessoas, tendo bons resultados nesse processo e indicando como uma forma viável de avaliar esse aspecto. Ainda, Yan (2010) analisou estilos cognitivos baseados no MBTI, visando indicar perfis que melhor se ajustariam ao aprendizado virtual e seus resultados permitiram a identificação das necessidades de aprendizagem e o ajuste do foco para estimular e promover a aprendizagem baseada em ambiente virtual e e-learning.

No Brasil, é possível perceber uma carência de estudos de validação do MBTI. Em pesquisa nas bases de dados de estudos sobre o MBTI com dados brasileiros de 1990 a 2010, foi encontrado somente dois artigos na base Lilacs e nenhum artigo nas bases BVS e Medline. Desses dois estudos identificados, um deles trata de evidenciar a relevância de classificação dos tipos junguianos no contexto organizacional a partir de considerações teóricas e apresentação do MBTI como instrumento de avaliação relevante dentro desse contexto (Nagelschmidt, 1993). O único estudo empírico identificado foi feito por Zacharias (1995) com policiais militares do Estado de São Paulo e analisou a predominância dos tipos psicológicos e as consequências para a instituição e comunidade. Os resultados revelaram que parte dos problemas de atuação desses profissionais na comunidade está associada aos tipos psicológicos deles, o que é compatível com os tipos junguianos, ressaltando a contribuição da tipologia para a área organizacional, especialmente na descrição dos perfis profissionais.

É possível observar que nenhum estudo analisou evidências para a estrutura interna desse instrumento, ressaltando a lacuna de literatura. Considerando a necessidade de estudos mais detalhados sobre a validade do MBTI para a população brasileira, este estudo se propõe então realizar um estudo da estrutura fatorial do MBTI.

\section{Método}

\section{Base de Dados}

Foi utilizado um banco de dados contendo descrições e dados para o MBTI. Estes foram coletados entre 2006 e 2009 e perfazem um total de 6.460 
protocolos de sujeitos com idades variando entre 14 e 77 anos $(M=37,20 ; D P=9,6)$, sendo $35,8 \%$ (1858) de sujeitos do sexo feminino.

\section{Instrumento}

O Myers-Briggs Type Indicator (MBTI) é um inventário que tem por objetivo avaliar a personalidade segundo a teoria dos tipos psicológicos de Jung de forma simples e sucinta. É um indicador de tipos psicológicos que foi desenvolvido com a finalidade de auxiliar as pessoas a identificar determinadas preferências pessoais significativas. O formulário $\mathrm{M}$ consiste de uma bateria de 93 perguntas ou itens com respostas dicotômicas, ou seja, admite apenas uma resposta de duas possíveis, que a pessoa que está sendo avaliada responde. Os itens estão divididos em quatro escalas de preferências bipolares e cada pergunta avalia uma preferência específica. Cada resposta conta um ponto para um polo ou outro dessa preferência. Assim, temos: funções da Percepção (sensação ou intuição): avaliada por 26 perguntas. Cada polo pode acumular um máximo de 26 pontos, se todas as respostas às 26 perguntas forem referentes àquele polo. Funções de Julgamento (pensamento ou sentimento): avaliada por 24 perguntas. Cada polo pode acumular um máximo de 24 pontos, se todas as respostas às 24 perguntas forem referentes àquele polo. Funções de Orientação (julgamento ou percepção): avaliada por 22 perguntas. Cada polo pode acumular um máximo de 22 pontos, se todas as respostas às 22 perguntas forem referentes àquele polo. Funções da Atitude (extroversão ou introversão): avaliada por 21 perguntas. Cada polo pode acumular um máximo de 21 pontos, se todas as respostas às 21 perguntas forem referentes àquele polo. Empregou-se a versão adaptada ao português proposta por Zacharias (1994).

\section{Procedimento}

Com o objetivo de explorar a estrutura interna da versão brasileira do inventário, foram feitas duas análises, a saber, uma análise fatorial confirmatória (AFC) (etapa 1) e uma análise fatorial exploratória com índices confirmatórios (AFE) (etapa 2). Para a estimação dos parâmetros da AFE com rotação oblimin, mais próxima do modelo confirmatório e precisão (alfa) foi usado o software $\mathrm{R}$ indicado para a estimativa da análise fatorial de itens dicotômicos a partir do cálculo de correlações tetracóricas (Damásio, 2012).

Utilizaram-se as análises de distância de Cook e de Mahalanobis para identificar problemas quanto à multicolinearidade e casos extremos uni e multivariados. Para a estimação dos parâmetros da AFC foi utilizado o MPLUS 15 e considerou-se a matriz de correlações tetracóricas como dados de entrada, tendo sido estimados os parâmetros por meio do método de Máxima Verossimilhança Robusta ou MLR (Maximum Likelihood Robust). Os seguintes índices de ajuste foram considerados (Byrne, 1989; Damásio, 2012; Tabachnick \& Fidell,
2001): 1. a razão $\chi^{2} / g l$ é considerada uma bondade de ajuste subjetiva, recomendando-se um valor próximo a 2 como evidência de adequação do modelo teórico; 2. o CFI e TLI (Comparison Fit Index, Tucker Lewis), em que valores próximos a 0,90 sugerem um ajustamento satisfatório; e 3. o RSMEA, com seu respectivo intervalo de confiança de $90 \%$, corresponde ao tamanho dos residuais que resultam quando o modelo é empregado para predizer os dados; valores inferiores a 0,05 indicam um bom ajustamento, sendo admitidos aqueles abaixo de 0,10 como expressando um ajustamento razoável.

\section{Resultados}

Os resultados foram divididos em duas seções. Inicialmente optou-se pelo modelo confirmatório para investigar se a estrutura inicial do instrumento proposto seria mantida no Brasil. Posteriormente com vista a identificar ajustes necessários, a análise exploratória foi conduzida.

\section{Análise fatorial confirmatória}

Com o objetivo de testar a consistência do modelo proposto teoricamente para o MBTI, foi realizada uma análise fatorial confirmatória. Antes disso, executou-se análises exploratórias para se verificar a adequação desse modelo aos dados em questão (Tabachnick \& Fidell, 2001). Inicialmente, as omissões não chegaram a $5 \%$ do total do arquivo e não foram identificados problemas quanto à multicolinearidade ou mesmo identificados casos extremos univariados ou multivariados que afetassem os resultados (informações obtidas pelas análises de distância de Cook e de Mahalanobis). Ainda, os valores de Kurtosis variaram de $-1,4$ a 0,05 e Skewness de 1,6 a $-1,80$. No entanto, somente dois itens (20 e 25) passaram dos limites de -1 e 1 em Skewness e 13 na Kurtosis. Assim, esses dados são moderadamente anormais, o que justificou o emprego do método da máxima verossimilhança na estimação dos parâmetros, conforme indicado por Arbuckle e Worthke (1999). Empregou-se o método de máxima verossimilhança robusta no software MPLUS.

Dessa forma, foi testado o modelo com quatro fatores quais sejam, funções de Julgamento, Atitudes, Percepção e Orientação, reunindo todos os 93 itens da versão brasileira da escala. Os parâmetros foram calculados a partir da matriz de correlações tetracóricas. Os índices da qualidade de ajustamento encontrados foram os seguintes: razão $\chi^{2} / g l=9,65, \mathrm{CFI}=0,73$, TLI $=0,72 \mathrm{e}$ RMSEA $=0,03$. Os índices indicam um ajuste pobre do modelo, levando à opção por um modelo exploratório nos dados para identificar a melhor estrutura subjacente aos mesmos.

\section{Análise fatorial exploratória}

Para se estimar a quantidade de fatores a serem mantidos na escala, optou-se pela análise paralela, já que 
esta indica a quantidade de fatores obtidos diferente do que pudesse ser obtida aleatoriamente. A Figura 1 apresenta a quantidade de fatores obtidos empirica e aleatoriamente, estabelecendo um comparativo. Por esses dados, identifica-se que até 10 fatores podem ser obtidos empiricamente não correndo risco de serem fatores aleatórios. Assim, restringiu-se os quatro fatores que mais faziam sentido teoricamente pela teoria junguiana que embasou a construção do MBTI. Em seguida, os itens foram submetidos à análise fatorial com correlações tetracóricas e rotação GEOMIN (oblíqua) com índices confirmatórios, $\chi^{2} / g l=61149.11 / 461$, TLI=0,90, RMSEA $=0,04$, usando-se o software R. Os índices confirmatórios foram melhores que os da análise confirmatória inicialmente apresentada, excetuando-se os valores de $\chi^{2} / g l$.

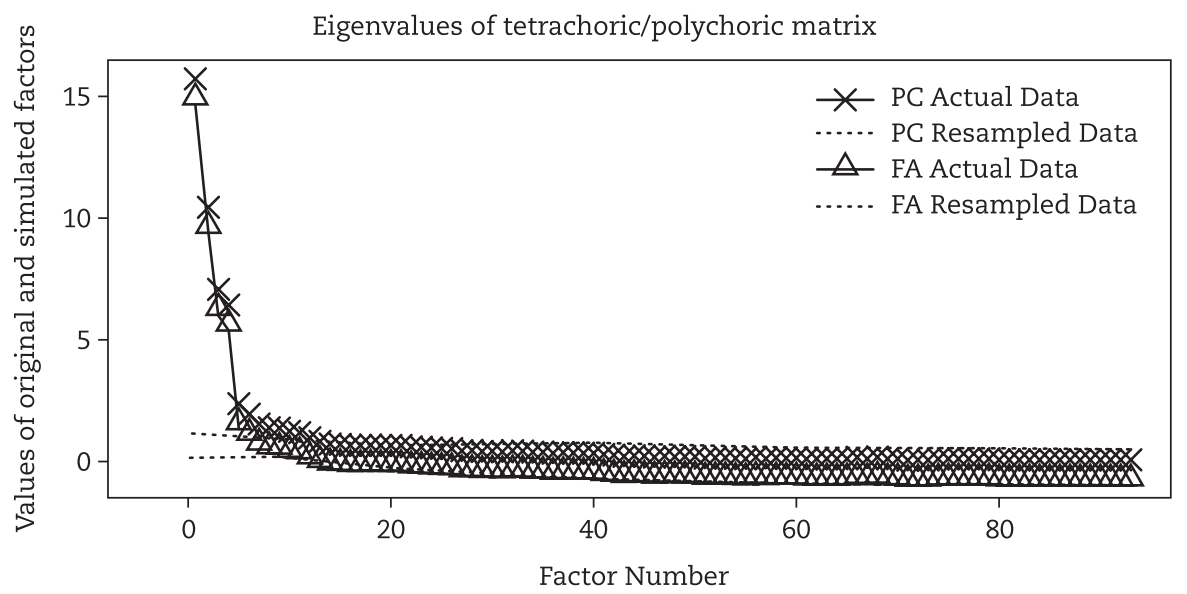

Figura 1. Gráfico de sedimentação com resultados da análise paralela

Os resultados encontrados, dispostos na Tabela 2, indicaram a existência de quatro fatores principais para o inventário. Foram retirados itens com cargas fatoriais abaixo de 0,40 . Pode-se observar que os fatores apresentam diferenças significativas na quantidade de variância explicada, sendo que o primeiro fator explica $11 \%$ da variância; o segundo fator explica $9 \%$; o terceiro fator, $10 \%$ e o quarto fator, $6 \%$. A quantidade total de variância explicada pelo modelo com quatro fatores é de 36\%. Na Tabela 2, são apresentadas, também, as cargas fatoriais dos itens para o modelo sugerido teoricamente com quatro fatores, foram excluídas cargas inferiores a 0,40 .

Como pôde ser observado, o fator 1 é composto pelos 11 itens da escala Atitudes. Todos os itens desse fator apresentaram cargas maiores que 0,50 e nenhum deles apresentou carga significativa em outros fatores o que possibilita a interpretação de sua robustez. O fator 2 , é composto pelos 8 itens pertencentes a escala de funções de Julgamento mas considerando os itens que não carregaram nos fatores ou que tiveram cargas fatoriais abaixo de 0,4. Nenhum item desse fator apresentou cargas significativas em outros fatores. O Fator 3 é formado pelos 10 itens da escala Orientação. O quarto fator foi composto pelos seis itens da escala de funções da Percepção. Nesse caso, todos os itens apresentaram cargas superiores a 0,40. Com isso, a escala final ficou composta por 35 itens.

O próximo passo foi analisar a fidedignidade do inventário e das quatro escalas que o compõe, para tanto, foi realizada a análise da consistência interna estimando os coeficientes do alfa de Cronbach. A precisão dos fatores foi obtida a partir da matriz de correlações tetracóricas (software $\mathrm{R}$ ). $\mathrm{O}$ valor do alfa para o inventário foi de 0,89 para os 35 itens finais. Esse valor é condizente com as exigências da comunidade científica acerca da consistência interna e está acima do mínimo exigido pelo Conselho Federal de Psicologia (CFP) que é de 0,60 ; o que sugere uma boa consistência interna do instrumento (Guilford \& Fruchter, 1978). O valor do alfa encontrado para a escala Atitudes foi de 0,76; na escala Orientação, 0,80; na escala Julgamento, 0,79 e na escala Percepção, 0,80 .

Tabela 2

Distribuição dos Itens do MBTI nos Quatro Fatores e Cargas Fatoriais após Rotação

\begin{tabular}{cccccc}
\hline & Atitude & Orientação & Julgamento & Percepção & $h^{2}$ \\
\hline 38V13 & 0,64 & $-0,09$ & $-0,08$ & 0,02 & 0,43 \\
91V34 & 0,64 & 0,06 & 0,08 & $-0,05$ & 0,41 \\
\hline
\end{tabular}


Tabela 2 (continuação) Distribuição dos Itens do MBTI nos Quatro Fatores e Cargas Fatoriais após Rotação

\begin{tabular}{|c|c|c|c|c|c|}
\hline & Atitude & Orientação & Julgamento & Percepção & $h^{2}$ \\
\hline 83V32 & 0,59 & $-0,07$ & $-0,06$ & 0,04 & 0,36 \\
\hline $85 V 33$ & 0,56 & 0,07 & 0,1 & $-0,11$ & 0,34 \\
\hline 42V14 & 0,56 & $-0,06$ & $-0,07$ & $-0,06$ & 0,35 \\
\hline $19 \mathrm{V7}$ & 0,55 & 0,08 & 0,05 & $-0,07$ & 0,31 \\
\hline 79V30 & 0,53 & 0,06 & 0,02 & 0,01 & 0,28 \\
\hline 07V3 & 0,53 & 0,05 & 0,07 & $-0,02$ & 0,28 \\
\hline $10 \mathrm{~V} 4$ & 0,53 & 0,03 & 0,04 & 0 & 0,28 \\
\hline 17V6 & $-0,08$ & 0,49 & $-0,09$ & $-0,01$ & 0,23 \\
\hline $73 \mathrm{~V} 27$ & $-0,08$ & $-0,06$ & $-0,04$ & 0,53 & 0,29 \\
\hline 28V11 & $-0,07$ & 0,62 & $-0,01$ & 0,11 & 0,43 \\
\hline $78 V 29$ & $-0,07$ & 0,48 & 0,04 & 0,02 & 0,26 \\
\hline 03V1 & 0,7 & $-0,06$ & $-0,07$ & 0,06 & 0,5 \\
\hline 26V10 & 0,7 & 0,01 & $-0,02$ & 0,04 & 0,49 \\
\hline $65 \mathrm{~V} 25$ & 0,05 & 0,06 & 0,12 & 0,61 & 0,43 \\
\hline $59 V 21$ & $-0,04$ & 0,58 & 0,07 & 0,05 & 0,39 \\
\hline $15 \mathrm{~V} 5$ & $-0,04$ & $-0,05$ & 0,55 & 0,02 & 0,3 \\
\hline $53 \mathrm{~V} 19$ & 0,04 & 0,02 & 0,48 & 0,1 & 0,25 \\
\hline $61 \mathrm{~V} 22$ & 0,04 & 0 & 0,54 & 0,12 & 0,32 \\
\hline $76 V 28$ & 0,03 & 0,68 & $-0,05$ & 0,01 & 0,44 \\
\hline $64 \mathrm{~V} 24$ & 0,03 & 0,61 & 0,03 & 0,01 & 0,39 \\
\hline 04V2 & 0,03 & 0,58 & $-0,11$ & 0,04 & 0,33 \\
\hline $20 \mathrm{~V} 8$ & 0,03 & 0,52 & $-0,01$ & $-0,05$ & 0,26 \\
\hline 50V18 & $-0,03$ & $-0,06$ & $-0,08$ & 0,56 & 0,3 \\
\hline $21 \mathrm{~V} 9$ & $-0,03$ & $-0,03$ & 0,65 & $-0,01$ & 0,42 \\
\hline 93V35 & 0,02 & 0,58 & 0,1 & $-0,01$ & 0,38 \\
\hline 80V31 & 0,01 & 0,67 & 0 & $-0,07$ & 0,43 \\
\hline $58 \mathrm{~V} 20$ & $-0,01$ & 0,04 & 0,64 & $-0,04$ & 0,42 \\
\hline $31 \mathrm{~V} 12$ & $-0,01$ & $-0,04$ & 0,68 & $-0,1$ & 0,45 \\
\hline $46 \mathrm{~V} 15$ & 0,01 & 0 & 0,01 & 0,55 & 0,3 \\
\hline $48 \mathrm{~V} 16$ & 0 & 0,08 & 0,07 & 0,59 & 0,4 \\
\hline $63 \mathrm{~V} 23$ & 0 & 0,04 & $-0,05$ & 0,57 & 0,33 \\
\hline 49V17 & 0 & 0,03 & 0,59 & 0,1 & 0,39 \\
\hline 70V26 & 0 & 0,03 & 0,65 & 0,03 & 0,44 \\
\hline
\end{tabular}

\section{Discussão}

A propósito da estrutura fatorial, os resultados do presente estudo apontaram para uma estrutura em quatro fatores com quantidade de itens reduzidos. Tal redução demonstrou melhorar o ajuste do modelo, já que foram usados somente os itens com maiores correlações e com cargas fatoriais mais elevadas. Esses achados, além de serem condizentes com as expectativas teóricas, já que avaliam as dimensões Disposição, Percepção, Julgamento e função preconizadas por Jung (2000), em termos de qualidade de ajuste estão em consonância com as sugestões para os índices de ajuste em estudos realizados internacionalmente, após a análise fatorial exploratória com índices confirmatórios (Jöreskog \& Sörbom, 1981). Poucos trabalhos, em âmbito internacional alcançaram parâmetros psicométricos adequados para esse instrumento, e a literatura tem enfatizado discrepâncias nesse particular (Folger et al., 2003; Salter, Forney, Evans, \&2005; Sullivan \& Hansen, 2004; Wheeler et al., 2004).

Assim, pesquisas, como as de Beuke t al. (2006), Deakin (2006), Hackston (2005), Harvey et al. (1995), Saggino e Kline (1995), Saggino et al. (2000), apesar de obterem índices satisfatórios no que se refere à consistência interna do MBTI, obtiveram parâmetros de até 0,70 , enquanto os dados da presente pesquisa indicaram valores de alfa acima de 0,80 , revelando uma maior adequação. Apesar de exibirem uma quantidade pequena de problemas quanto à correlação item total, os itens da escala demonstraram índices elevados de consistência interna, superiores aos estudos internacionais com 
esse instrumento, sugerindo boa precisão dessa escala na amostra brasileira.

Também, a variabilidade da amostra em termos de escores extremos foi alta apesar da característica dicotômica do instrumento, o que implementou uma das limitações de uma série de estudos da literatura internacional sobre o MBTI em que a análise da sua estrutura interna em grupos com pouca concentração de sujeitos em escores extremos não fornecia resultados tão satisfatórios (Gardner \& Martimko, 1996). Apesar disso, entende-se que a variabilidade excessiva de faixas etárias da amostra pode impor limitações quanto ao formato e adequação da linguagem dos itens para diferentes faixas etárias. Optou-se por adultos para assegurar uma melhor compreensão, todavia, para idosos, a linguagem pode ter sido uma barreira. Apesar disto, evidenciou-se uma estrutura fatorial mais enxuta com menos itens (o que pode ter também decorrido de má compreensão por parte dos mais velhos, sobretudo, afetando a magnitude das correlações de alguns itens) com bons índices de ajuste. Gardner e Martimko (1996) e outros como de Pittenger (1993) sugerem que a o formato dicotômico das respostas evidenciam uma série de dificuldades na classificação dos tipos de personalidade, como na análise fatorial em que muitas vezes as estruturas encontradas não são consistentes com a teoria junguiana. Nesse sentido, a presente pesquisa, executada com uma amostra maior, produziu mais variância para cada uma das medidas, superando a limitação do formato das respostas, ao menos em certa medida, evidenciando uma estrutura fatorial condizente teoricamente e com bons índices de ajuste. Apesar disso, por se tratar de uma questão recorrente na literatura e por nem sempre se dispor de amostras altamente representativas dos traços em questão, outros formatos de resposta como o Likert poderiam ser aventados e estudados quanto à sua adequação por meio de análise de créditos parciais no modelo de Rasch, por exemplo (Bartholomeu, Montiel, \& Machado, 2013).

Em termos de conclusão, espera-se, com isso, que este estudo favoreça o desenvolvimento de novas investigações com esse instrumento, catalisando a pesquisa nesse particular e a implementação do teste. Por fim, o modelo de Rasch também poderia ser viável por fornecer outras evidências quanto à estrutura interna desse instrumento. Tal aspecto convida a novas pesquisas. Outro aspecto indicado na literatura sobre o MBTI e não analisado no presente trabalho seriam os pontos de corte quanto às classificações nos tipos de personalidade (Pittenger, 1993), restando a sugestão.

\section{Referências}

Arbuckle, J., \& Wothke, W. (1999). AMOS 4 user's reference guide. Chicago: Smallwaters Corp.

Bartholomeu, D., Montiel, J. M., \& Bernstein, M. J. (2014). Social skills and study group acceptance: Research with higher education. International Journal of Advances in Psychology, 3(4), 111-119.

Bartholomeu, D., Montiel, J. M., \& Machado, A. A. (2013). Otimização da escala dos itens do CSAI em atletas. Interação em Psicologia (Impresso), 17, 77-87.

Bartholomeu, D., Montiel, J. M., \& Pessotto, F. (2012). Sociometria e habilidades sociais em alunos do ensino médio. Revista Estudos Interdisciplinares em Psicologia, 2(2), 211-228.

Bento, W. T., Bartholomeu, D., Montiel, J. M., \& Machado, A. A. (2013). Avaliação da relação entre ansiedade e componentes sociométricos em atletas do handball. Coleção Pesquisa em Educação Física, 12(2), 41-31.

Beuke, C. J., Freeman, D. G., \& Wang, S. (2006). Reliability and validity of the Myers-Briggs Type Indicator ${ }^{\circledR}$ Form M when translated into traditional and simplified Chinese characters. Paper apresentado no fifth Psychological Type and Culture - East and West: A Multicultural Research Conference, Honolulu, Hawaii. Recuperado de http://typeandculture.org/Pages/C_papers06/BeukeChineseRelib.doc.pdf

Briggs Myers, I. Kirby, L. K., \& Myers, I. D. (1998). Introduction to type: A guide to understanding your results on the Myers-Briggs Type Indicator. CPP, Inc. Palo Alto, California.

Byrne. R. M. J. (1989). Suppressing valid inferences with conditionals. Cognition, 31(1), 61-83.

Cohen, Y., Ornoy, H., \& Keren, B. (2013). MBTI personality types of project managers and their success: A Field Survey. Project Management Journal 44(3), 78-87.

Damásio, B. F. (2012). Uso da análise fatorial exploratória em psicologia. Avaliação Psicológica, 11(2), 213-228.

Deakin, P. (2006). German MBTI ${ }^{\oplus}$ Step $I^{\mathrm{TM}}$ data supplement. Oxford, England: OPP, Ltd.

Folger, W. A., Kanitz, H. E., Knudsen, A. E., \& McHenry, S. (2003). Analysis of MBTI type patterns in college scholars. College Student, 37(4), 598-603.

Gardner, W. L., \& Martinko M. J. (1996). Using the Myers-Briggs type indicator to study managers. A Literature Review and Research Agenda Journal of Management 22(1), 45-83.

Guilford, J. P., \& Fruchter, B. (1978). Fundamental statistics in psychology and education. New York: McGraw-Hill.

Hackston, J. (2005). French MBTI ${ }^{\circledast}$ Step I Data Supplement. Oxford: OPP Ltd.

Harvey, R. J., Murry, W. D., \& Stamoulis, D. T. (1995). Unresolved issues in the dimensionality of the Myers-Briggs type indicator. Educational and Psychological Measurement, 55(4), 535-544.

Jöreskog, K. G., \& Sörbom, D. (1981). Lisrel V. Chicago: International Educational Services.

Jung, C. G. (1928/1971). O eu e o inconsciente. Petrópolis: Vozes.

Jung, C. G. (1964). Man and his symbols. New York: Dell. 
Jung, C. G. (2000). Os arquétipos e o inconsciente coletivo. Petrópolis: Vozes.

Lee, K. H., Choi, Y., \& Stonier, D. J. (2012). Evolutionary algorithm for a genetic robot's personality based on the Myers-Briggs type indicator. Robotics and Autonomous Systems, 60(7), 941-961.

Lessa, E. M. (2003). Equipes de alto desempenho: a tipologia de Jung nas organizações. São Paulo: Vetor.

Nagelschmidt, A. M. P. C. (1993). O uso dos tipos psicológicos junguianos no contexto das organizações. Boletim de Psicologia: Instituto de Psicologia da USP, 43(98/99), 81-94.

Pasquali, L. (1997). Psicometria: teoria e aplicações. Brasília-DF: Editora UnB.

Pasquali, L. (2003). Os tipos humanos: a teoria da personalidade. Petrópolis: Vozes.

Pittenger, D. J. (1993), Measuring the MBTI and coming up short. Journal of Career Planning and Employment, 54, 48-53.

Ramos, L. M. A. (2005). Os tipos psicológicos na psicologia analítica de Carl Gustav Jung e o inventário de personalidade Mayers-Briggs type indicator (MBTI): contribuições para a psicologia educacional, organizacional e clínica. Educação Temática Digital, 6(2), 137-180.

Rien, S, Marian, P., \& Helen, S. (2010, September). The use of MBTI in software engineering. 22nd Annual Psychology of Programming Interest Group, Universidad Carlos III de Madrid, Madrid, Espanha.

Saggino, A., \& Kline, P. (1995). Item factor analysis of the Italian version of the Myers-Briggs type indicator. Personality and Individual Differences, 19(2), 243-249.

Saggino, A., Cooper C., \& Kline, P. (2000). A confirmatory factor analysis of the Myers-Briggs type indicator. Personality and Individual Differences, 30(1), 3-9.

Salter, D. W., Forney, D. S., \& Evans, N. J. (2005). Two approaches to examining the stability of Myers-Briggs type indicator scores. Measurement and Evaluation in Counseling and Development, 37(4), 208-219.

Schaubhut, N. A., \& Thompson, R. (2010). Technical brieffor the MBTI ${ }^{\circledR}$ Form M and Form Q Assessments-Simplified Chinese. Mountain View, CA: CPP, Inc.

Schaubhut, N. A., Herk, N. A., \& Thompson, R. C. (2009). MBTI ${ }^{\circledast}$ Form M manual supplement. Mountain View, CA: CPP, Inc.

Sullivan, B. A., \& Hansen, J. C. (2004). Mapping associations between interests and personality. Journal of Counseling Psychology, 51(3), $287-298$.

Tabachnick, B. G., \& Fidell, L. S. (2001). Using multivariate analysis. Boston: Allyn and Bacon.

Taylor, N., \& Yiannakis, C. (2007). South African MBTI ${ }^{\circledast}$ Form M data supplement. Johannesburg, South Africa: Jopie Van Rooyen \& Partners, S.A. (Pty) Ltd.

Wheeler, P., Hunton, J., \& Bryant, S. (2004). Accounting information systems research opportunities using personality type theory and the Myers-Briggs type indicator. Journal of Information Systems, 18(1), 1-19.

Yan, S. (2010, June). Successful implementation of e-learning from the situated cognition and MBTI perspective. Paper apresentado na International Conference on. Qinhuangdao, China. Recuperado de http://ieeexplore.ieee.org/xpl/login.jsp?tp =\&arnumber $=5541142 \& u r l=h t t p \%$ 3A\%2F\%2Fieeexplore.ieee.org\%2Fxpls\%2Fabs_all.jsp\%3Farnumber\%3D5541142

Yilmaz, M., \& O'Connor, R. V. (2012, September). Towards the understanding and classification of the personality traits of software development practitioners: Situational context cards approach. Software Engineering and Advanced Applications 2012 38th EUROMICRO Conference. IEEE, (pp. 400-405). Cesme, Izmir.

Yolles, M., \& Fink, G. (2009). Migrating personality theories Part 2: Towards a theory of the balanced personality? Kybernetes, 38(9), 14611490.

Zacharias, J. J. M. (1995). Tipos psicológicos junguianos e escolha profissional: uma investigação com policiais militares da cidade de São Paulo. São Paulo: Vetor.

Ziegert, A. L. (2000). The role of personality temperament and student learning in principles of economics: Further evidence. The Journal of Economic Education, 31(4), 307-322.

\section{Sobre os autores}

Gleiber Couto é psicólogo e doutor em Psicologia na área de Avaliação Psicológica pela Universidade São Francisco. Atualmente é professor da Universidade Federal de Goiás na regional de Catalão.

Daniel Bartholomeu é psicólogo e doutor em psicologia na área de Avaliação Psicológica pela Universidade São Francisco. Atualmente é professor do Centro Universitário Fundação Instituto para o Ensino de Osasco, no programa de Pós-Graduação Strictu Sensu em Psicologia Educacional e atua como psicólogo clínico.

José Maria Montiel é psicólogo e doutor em psicologia na área de Avaliação Psicológica pela Universidade São Francisco. Atualmente é professor do programa de Pós-Graduação Strictu Sensu em Psicologia Educacional no Centro Universitário Fundação Instituto de Ensino para Osasco (UniFIEO/SP). 